\title{
Fetus Small for Gestational Age with Abdominal Circumference Less than Tenth Percentile
}

National Cancer Institute

\section{Source}

National Cancer Institute. Fetus Small for Gestational Age with Abdominal Circumference Less than Tenth Percentile. NCI Thesaurus. Code C114878.

A fetus that does not grow beyond the 10th percentile of conventionally accepted weight for gestational age and whose abdominal circumference falls below the tenth percentile. 